\title{
DIETA Y VARIACIÓN MORFOLÓGICA CRANEOFACIAL EN POBLACIONES HUMANAS DEL GOLFO SAN MATÍAS (PATAGONIA, ARGENTINA)
}

\author{
VIRGINIA A. COBOS*, CRISTIAN M. FAVIER DUBOIS"* \& S. IVAN PEREZ
}

\begin{abstract}
RESUMEN
El objetivo de este trabajo es estudiar la variación morfológica craneofacial de las poblaciones humanas que habitaron la costa rionegrina del golfo San Matías (Patagonia, Argentina), y áreas vecinas, durante el Holoceno tardío (3.000-400 años AP) y su relación con cambios en la dieta. El registro arqueológico de esta región se caracteriza por presentar información detallada de los contextos ecológicos que permite establecer cambios en la dieta a partir de datos isotópicos y arqueológicos, y un control de la variable cronológica mediante fechados radiocarbónicos. En particular exploramos, mediante el empleo de técnicas de la morfometría geométrica en 2D, una hipótesis que postula la existencia de una relación entre la variación en la morfología craneofacial y los cambios en la dieta ocurridos alrededor de ca. 1.600 años AP en el área. Con este fin se analizó el patrón de cambio morfológico en el modulo facial y la base del cráneo en las poblaciones procedentes de las regiones del golfo San Matías, de la costa norte rionegrina, del valle inferior del río Chubut y de San Blas e Isla Gama, para las cuales se ha postulado la presencia de grados variables de diferencias en la dieta entre los períodos señalados. Nuestros resultados muestran que el patrón de variación morfológica craneofacial observado en el noreste de Patagonia en general, y en el área del golfo de San Matías en particular, es complejo, caracterizado por un comportamiento diferencial de las dos regiones anatómicas estudiadas (el esqueleto facial y la base del cráneo) en relación con la variación ecológica.
\end{abstract}

PALABRAS CLAVE: bioarqueología, dieta, golfo de San Matías, morfometría geométrica.

$$
\begin{aligned}
& \text { DIET AND CRANIOFACIAL MORPHOLOGICAL VARIATION IN HUMAN } \\
& \text { POPULATIONS OF THE SAN MATİAS GULF (PATAGONIA, ARGENTINA) }
\end{aligned}
$$

\section{ABSTRACT}

The aim of this paper is to study the craniofacial morphological variation of human populations that inhabited the San Matías gulf coast (Río Negro, Patagonia, Argentina) and neighboring areas during

División Antropología. Facultad de Ciencias Naturales y Museo. Universidad Nacional de La Plata. La Plata (1900), Argentina. Consejo Interuniversitario Nacional (CIN). Argentina. virginiacobos@gmail.com

* INCUAPA. Olavarria (7400), Argentina. Consejo Nacional de Investigaciones Científicas y Técnicas (CONICET). Argentina. cfavier3@gmail.com

** División Antropología. Facultad de Ciencias Naturales y Museo. Universidad Nacional de La Plata. La Plata (1900), Argentina. Consejo Nacional de Investigaciones Científicas y Técnicas (CONICET). Argentina. ivanperezmorea@gmail.com 
the late Holocene (3000-400 years BP), and its relationship to changes in the diet. The archaeological record of this region is characterized by detailed information of the ecological contexts that allows to differentiate changes in the diet from isotopic and archaeological data. The chronology for this area has been well established by radiocarbon dating. In particular, by using geometric morphometrics techniques in $2 \mathrm{D}$, we explore a hypothesis that postulates the existence of a relationship between the variation in craniofacial morphological and dietary changes that occurred around ca. 1.600 years AP in the area. Here, we analyzed the pattern of morphological change in the facial module and cranial base in populations from the San Matías gulf, the northern coast of Negro river, the lower Chubut river valley, and San Blas and Isla Gama, for which the presence of varying degrees of differences in diet between the periods studied has been indicated. Our results show that the pattern of craniofacial morphological variation observed in northeastern Patagonia, in general, and in the area of San Matías gulf, in particular, is complex and characterized by a different behavior of the two anatomical regions studied (the facial skeleton and cranial base) relative to ecological variation.

KEY WORDS: bioarchaeology, diet, San Matías gulf, geometric morphometrics.

\section{INTRODUCCIÓN}

El origen y mantenimiento de los patrones de variación fenotípica a nivel intra e interpoblacional puede resultar, en parte, de la influencia de diversos factores ecológicos (Badyaev, 2005; Carroll et al., 2007; Katzmarzyk \& Leonard 1998; Reznick, et al. 1997; Roseman, 2004; Schluter, 2000). Estos factores actúan sobre la variación fenotípica, tanto como un agente de selección favoreciendo determinadas variantes genéticas en distintos contextos ecológicos (Diniz-Filho et al. 2009; Katzmarzyk \& Leonard 1998; Schluter, 2000), como induciendo cambios a lo largo de la ontogenia debido a la capacidad de los genotipos para producir diferentes fenotipos dependiendo del ambiente en el que se desarrolla el organismo, e.g. plasticidad fenotípica (Bogin \& Rios, 2003; Carroll et al., 2007; Nijhout, 2003; Perez \& Monteiro, 2009; Pigliucci, 2001; Pucciarelli \& Oyhenart 1987; West-Eberhard, 2003).

Diversos estudios realizados sobre poblaciones humanas de Sudamérica han señalado la contribución de factores ecológicos tales como la dieta y la temperatura en la estructuración de la variación en el tamaño de diversas unidades esqueletales y en la forma del esqueleto craneofacial (Bernal et al., 2006; Bernal et al., 2010; Béguelin, 2010; González-José et al., 2005; Perez \& Monteiro 2009; Perez et al., 2011; Rothammer \& Silva 1990; Sardi \& Ramirez-Rozzi, 2005). Estos estudios se han focalizado, principalmente, en la descripción de la variación morfológica en escala geográfica continental y supra-regional, así como en una escala temporal acotada (i.e., unas pocas generaciones). En conjunto, estos trabajos han sugerido que la influencia de los factores ecológicos sobre la variación fenotípica entre poblaciones humanas resulta variable a través de distintas escalas espaciales. En éste sentido, el análisis de los patrones de variación a escalas espaciales menores -microrregional y/o local-, resulta escaso y se ha limitado a áreas geográficas particulares y escalas temporales relativamente amplias (ca. 7.000 años; e.g. Cocilovo et al., 2001; Perez et al., 2009).

En este trabajo se estudia la variación morfológica craneofacial de las poblaciones humanas que habitaron la costa rionegrina del golfo San Matías (Patagonia, Argentina) durante el Holoceno tardío (3.100-400 años AP) y su relación con cambios en la dieta. El registro arqueológico de esta región se caracteriza por presentar información detallada de los contextos ecológicos que permite establecer cambios en la subsistencia a partir de datos isotópicos (i.e. paleodietas a partir de $\delta 13 \mathrm{C}$ y $\delta 15 \mathrm{~N}$ sobre colágeno) y arqueológicos (e.g. tecnología lítica, restos arqueofaunísticos y del procesamiento de vegetales) y un control de la variable cronológica mediante fechados radiocarbónicos que permiten determinar confiablemente la antigüedad de las muestras óseas analizadas (Favier Dubois et al., 2009; Favier Dubois \& Scartascini, 2012). Por lo tanto, esta región provee una oportunidad única para explorar la relación entre variación morfológica y dieta en una escala temporal acotada y microrregional. 
Los trabajos efectuados en el área indican que entre 2.200 y 1.600 años AP se produjo un cambio en la dieta de las poblaciones humanas prehistóricas que habitaron la costa rionegrina del golfo San Matías (Fig. 1). Los datos de isótopos estables $(\delta 13 \mathrm{C}$ y $\delta 15 \mathrm{~N})$, así como los conjuntos arqueofaunísticos recuperados en los sitios del área, muestran que hacia finales del Holoceno tardío se habría producido una reducción en la proporción de los ítems marinos consumidos y una diversificación en los recursos explotados, diferenciándose claramente tendencias temporales (Favier Dubios et al., 2009). En particular, Favier Dubois et al. (2009) han sugerido la existencia de dos periodos sucesivos con ocupación humana para el golfo de San Matías, que van de un uso intensivo a uno moderado de los recursos costeros (ver también Favier Dubois \& Scartascini 2012): 1) Uso intensivo (ca. 5.000-2.200 años AP). Se evidencia en los sitios el consumo de peces, mamíferos marinos, moluscos, crustáceos y aves marinas, así como la utilización de tecnologías de pesca vinculadas con el uso de redes. Se observa una baja diversidad artefactual de los conjuntos líticos, patrón consistente con una disminución del rango de especies explotadas en este período, mientras que con posterioridad al ca. 1.500 años $\mathrm{AP}$ la diversidad se incrementa. En este sentido la conspicua presencia de otolitos y de variadas pesas líticas puede ser considerada indicador de una forma de especialización costera, tratándose de grupos principalmente pescadores y marisqueadores (Favier Dubois \& Scartascini, 2012; Scartascini, Charo \& Volpedo, 2009). 2) Uso "moderado" (ca. 1.500-420 años AP). En los sitios correspondientes a este período se encuentra fauna marina similar respecto al período anterior, a la que se suman restos de guanaco, rhéidos y pequeños mamíferos tales como armadillos consumidos en proporciones antes no evidenciadas. Estos ítems se hallan asociados a tecnologías no registradas en los sitios del período anterior tales como cerámica y puntas de proyectil pequeñas (asociadas al uso de arco y flecha). Asimismo, se observa una alta frecuencia de artefactos de molienda para alimentos de origen vegetal, mientras que aquellos vinculados con la pesca disminuyen notablemente su señal. Para el siglo XVIII las crónicas históricas indican ya un abandono de la costa en el marco de los profundos

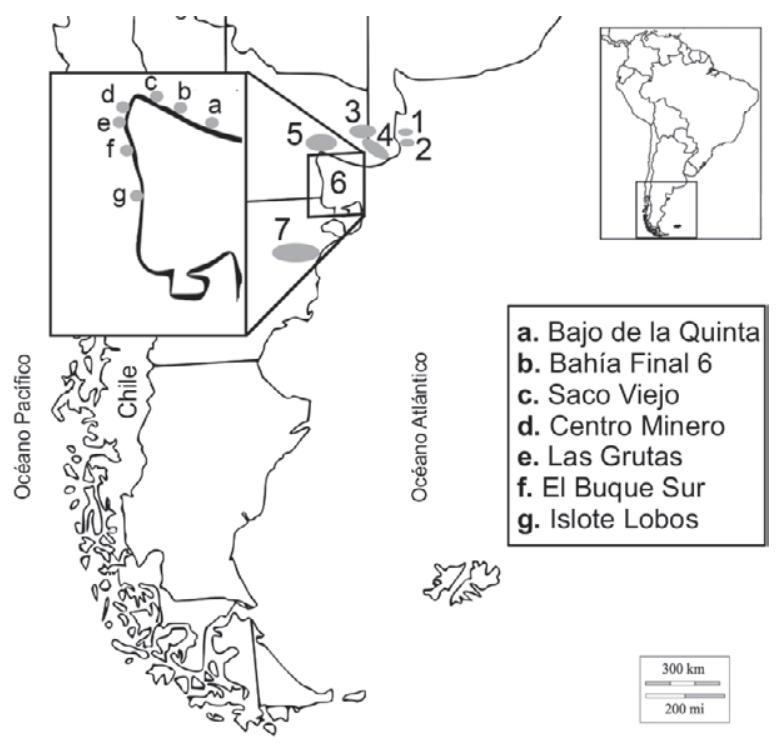

Fig. 1. Localización geográfica de las muestras analizadas: 1. Isla Gama, 2. San Blas, 3. Laguna del Juncal, 4. Valle inferior del Río Negro, 5. San Antonio Oeste, 6. Golfo San Matías, 7. Valle inferior del Río Chubut.

cambios ocurridos luego de la conquista española.

En este contexto, se explorará la hipótesis referida a la existencia de una influencia significativa de los cambios en la dieta ocurridos antes de ca.1.600 años AP en las poblaciones del área sobre el patrón temporal de variación morfológica craneofacial. Con fines comparativos también se analizará el patrón de variación morfológica en las poblaciones procedentes de las regiones de la costa norte rionegrina, del valle inferior del río Chubut, de San Blas y de la Isla Gama, para las cuales se ha postulado la presencia de grados variables de diferencias en la dieta entre los períodos señalados más arriba (Flensborg, 2012; Gómez-Otero, 2007; Martinez, 2008-2009). Debido a que el cráneo es una estructura diferenciada en módulos menores, en este trabajo se estudiarán dos regiones del cráneo, la base y la región facial, que han demostrado responder de manera diferencial a la influencia del ambiente (Harvati \& Weaver, 2006; Perez \& Monteiro, 2009). En particular, la base del cráneo alcanza su tamaño y forma adulto durante la ontogenia temprana del individuo y por lo tanto está poco expuesta a la influencia del ambiente, mientras que el esqueleto facial termina su crecimiento cuando el individuo alcanza la adultez y entonces la influencia del ambiente puede 
ser más importante (Perez \& Monteiro, 2009). La variación morfológica de estas estructuras será estudiada mediante el empleo de técnicas de la morfometría geométrica (Adams et al. 2004; Mitteroecker \& Gunz 2009).

\section{MATERIALES Y MÉTODOS}

\section{Composición de las muestras}

La muestra analizada en el presente estudio incluyó un total de 15 cráneos pertenecientes a la región rionegrina del golfo de San Matías rescatados en el marco de trabajos arqueológicos llevados a cabo por el equipo de investigación de uno de los autores (CMFD), de los que se cuenta con información contextual, fechados radiocarbónicos y análisis de isótopos estables ( $\delta 13 \mathrm{C}$ y $\delta 15 \mathrm{~N})$. Con fines comparativos, se incorporaron a la muestra 12 individuos provenientes de sitios de la región de San Antonio Oeste excavados a inicios del siglo XX, que no presentan información con la misma calidad que los recuperados recientemente. Asimismo, se analizaron 131 cráneos pertenecientes a individuos adultos de ambos sexos, procedentes de sitios arqueológicos de la región del valle inferior del río Negro, la laguna del Juncal, el valle inferior del río Chubut, la Isla Gama y la bahía San Blas (Tabla 1; Fig. 1). La asignación cronológica fue efectuada en estos casos a partir de fechados radiocarbónicos, el tipo de modificación craneana artificial e información contextual (Bernal et al., 2008; Favier Dubois et al., 2009; Perez, 2007). Con base en nuestras hipótesis y los estudios previos discutidos más arriba, la muestra fue subdividida en dos grupos cronológicos, uno que abarca desde el 3.100 al 2.000 años AP, y otro desde el 1.500 al 400 años AP.

La muestra procedente de la región del golfo de San Matías se encuentra depositada temporariamente en los laboratorios de la Unidad Ejecutora CONICET-INCUAPA (Investigaciones Arqueológicas y Paleontológicas del Cuaternario Pampeano) de la Universidad Nacional del Centro de la Provincia de Buenos Aires. Asimismo, las muestras procedentes de San Antonio Oeste, San Blas, Isla Gama, laguna del Juncal, valle inferior del rio Negro y de la región del valle inferior del río Chubut, seleccionadas con fines comparativos, fueron subdivididas temporalmente siguiendo los mismos criterios que la muestra procedente de la región del golfo de San Matías. Estas muestras se encuentran depositadas en la División Antropología de la Facultad de Ciencias Naturales y Museo de la Universidad Nacional de La Plata y en el Museo Etnográfico "J. B. Ambrosetti" de la Facultad de Filosofía y Letras de la Universidad de Buenos Aires.

La estimación de la edad y el sexo de los individuos se realizó siguiendo los procedimientos

Tabla 1. Muestra estudiadas en este trabajo.

\begin{tabular}{|c|c|c|c|}
\hline Sigla & Muestra & Período & $\mathbf{N}$ \\
\hline 1 & Isla Gama & $1500-400$ & 12 \\
\hline 2 & San Blas & $1500-400$ & 24 \\
\hline 3 & Laguna del Juncal & $3100-2000$ & 27 \\
\hline \multirow{2}{*}{4} & \multirow{2}{*}{ Valle inferior del Río Negro } & $1500-400$ & 18 \\
\hline & & $3100-2000$ & 16 \\
\hline \multirow{2}{*}{5} & \multirow{2}{*}{ San Antonio Oeste } & $1500-400$ & 7 \\
\hline & & $3100-2000$ & 6 \\
\hline \multirow{2}{*}{6} & \multirow{2}{*}{ Golfo San Matías } & $1500-400$ & 6 \\
\hline & & $3100-2000$ & 9 \\
\hline \multirow{2}{*}{7} & \multirow{2}{*}{ Valle inferior del Río Chubut } & $1500-400$ & 16 \\
\hline & & $3100-2000$ & 16 \\
\hline
\end{tabular}


estándar descriptos por Buikstra y Ubelaker (1994), utilizando rasgos de la pelvis y el cráneo cuando ambas estructuras anatómicas estaban disponibles. Sin embargo, dado que la mayor parte de los individuos se encuentran representados sólo por cráneos, las estimaciones

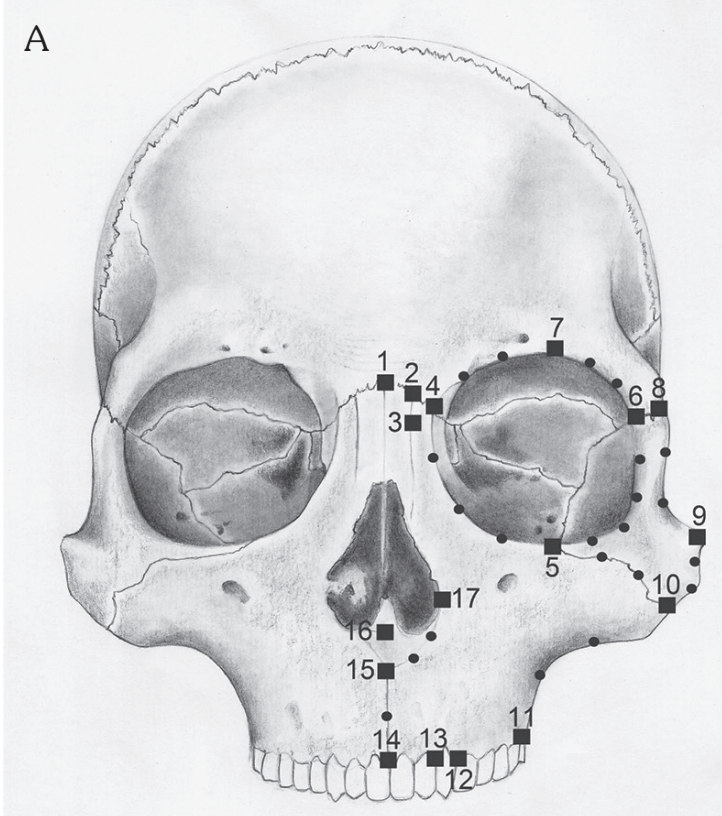

B

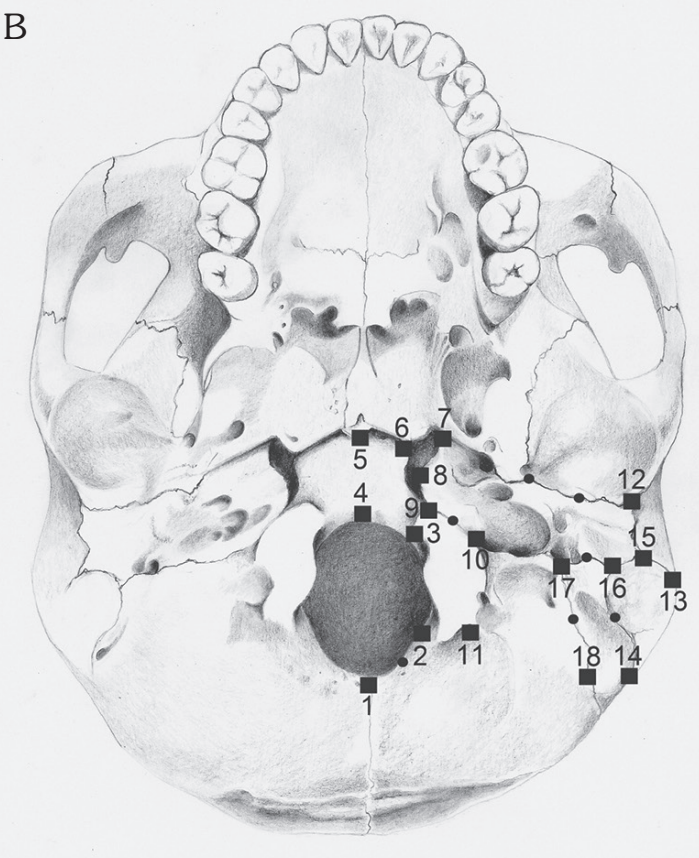

se efectuaron principalmente con rasgos craneales. Dado que algunos los rasgos craneales utilizados en los análisis morfométricos son usados para la estimación de sexo (Buikstra \& Ubelaker, 1994) y debido al tamaño de las muestras estudiadas, ambos sexos fueron combinados para los análisis morfométricos; sin embargo, se controló que los individuos masculinos y femeninos estuvieran representados en un número similar para cada una de las muestras de las diferentes regiones y grupos cronológicos. Asimismo, sólo individuos adultos, con la sutura esfeno-basilar obliterada y el tercer molar erupcionado, fueron utilizados en los análisis morfométricos.

\section{Análisis morfométricos}

Los análisis craneométricos se efectuaron empleando técnicas de la morfometría geométrica basadas en puntos anatómicos (landmarks) y contornos (semilandmarks) registrados sobre dos regiones del cráneo, la base y la región facial, que han demostrado responder de manera diferencial a la influencia del ambiente (Fig. 2; Harvati \& Weaver, 2006; Perez \& Monteiro, 2009).

Se tomaron fotos digitales en norma frontal y basal de todos los cráneos estudiados empleando una cámara Olympus SP 350. Se relevaron coordenadas de 17 landmarks y de 22 semilandmarks en 2D a partir de las imágenes en norma frontal (Fig. 2A), y de 18 landmarks y de 7 semilandmarks sobre las de norma basal (Fig. 2B), usando el programa tpsDIG2 versión 2.16 (Rohlf, 2012). El registro de las variables fue realizado por un único observador (VAC) con el propósito de eliminar el error interobservador. Se realizó la

Fig. 2. Landmarks (•) y semilandmarks (•) de la region facial (A) y basal (B) utilizados: A) Landmarks: 1.Nasion, 2.Nasofrontal, 3.Nasomaxilar, 4. Dacryon, 5.Zygoorbital, 6.Frontomalar anterior, 7.Supraorbital, 8.Frontomalar temporal anterior, 9. Zygion, 10. Zygomaxilar anterior, 11.Ectomolar, 12.Canino, 13.Incisivo, 14.Prostion, 15.Nasospinal, 16.Subespinal, 17.Alar ; B) Landmarks: 1. Opisthion, 2. Foramen lateral posterior, 3. Foramen lateral anterior, 4. Basion, 5. Esfenooccipital, 6. Esfeno-occipital lateral, 7. Petroso extremo anterior, 8. Petroso extremo posterior, 9. Condilar anterior, 10. Cóndilo lateral, 11. Condilar posterior, 12. Auricular anterior, 13. Mastoide anterior, 14. Mastoide posterior, 15. Auricular posterior, 16. Mastoide anterior, 17. Temporooccipital anterior, 18. Temporo-occipital posterior. 
estimación de la naturaleza y magnitud del error en el registro de las coordenadas de los landmarks y de los contornos descriptos por los semilandmarks, que puede afectar apreciablemente los resultados de los análisis morfométricos, mediante el empleo de ANOVA de medidas repetidas y correlación intraclase (Bernal et al., 2004). Los resultados obtenidos señalan bajos niveles de error intraobservador para ambos conjuntos de datos.

A partir de las coordenadas originales de landmarks y semilandmarks y mediante el empleo del Análisis Generalizado de Procrustes (Generalized Procrustes Analysis; Rohlf \& Slice, 1990) se obtuvieron las coordenadas superpuestas o variables de forma. Este método elimina las diferencias entre las configuraciones de puntos que son debidas a localización, escala y orientación original de los puntos. Los semilandmarks fueron deslizados a lo largo de sus respectivos contornos empleando el criterio de mínima distancia Procrustes (Bookstein et al., 2002; Perezet al., 2006). Posteriormente, a partir de las coordenadas superpuestas se calcularon componentes principales (CPs) y variables canónicas (VCs) para analizar el patrón de variación inter e intramuestral en la forma facial y de la base. Finalmente, se estableció la importancia de las diferencias observadas entre las muestras empleando un análisis multivariado de la varianza (MANOVA) sobre los CPs de todos los individuos que resumen ca. $90 \%$ de la variación total. Todos los análisis fueron efectuados utilizando los programas tpsRelw 1.49 (Rohlf, 2012), MorphoJ 1.03c (Klingenberg, 2011) y R 2.13.0 (R Development Core Team, 2012).

\section{RESULTADOS}

A lo largo del primer CP (CP 1) de la región facial la principal diferencia morfológica entre las muestras se observa en la robustez del malar (Fig. 3). Los individuos pertenecientes a las muestras ubicadas hacia los valores negativos de este primer eje presentan malares más robustos. En relación a la órbita, se observa un mayor tamaño relativo hacia el extremo de los valores positivos. A lo largo del segundo eje (CP 2) de la región facial, las muestras ubicadas en el extremo de los valores positivos presentan malares más robustos, así como órbitas relativamente más grandes que aquellos ubicados en el extremo de los valores negativos (Fig. 3). De este modo, los individuos de las muestras del extremo positivo de este eje presentan una región facial más amplia y robusta, es decir, de igual altura pero de mayor ancho relativo.

$\mathrm{Al}$ observar los wireframes obtenidos mediante el análisis de las variables canónicas, se observa que a lo largo del primer eje de las VC (VC 1) las principales diferencias en la región facial se dan en su altura relativa, presentando una región facial relativamente más alta aquellos individuos ubicados en el extremo negativo del eje (Fig. 4). Con respecto al malar, a diferencia de lo observado para el análisis de los componentes principales, se observa un mayor tamaño relativo de este hueso hacia los valores positivos. La morfología de la órbita varía en el mismo sentido que el observado para el análisis anterior. En el segundo eje (VC 2), el tamaño relativo del hueso malar varía en el mismo sentido que lo observado para el primer eje, inversamente a lo ocurrido con el tamaño relativo de las órbitas. A su vez, los individuos ubicados hacia el extremo de los valores positivos, presentan una región facial relativamente más baja (Fig. 4).

Cabe destacar el hecho de que las muestras que pertenecen al primer período temporal se diferencian de aquellas correspondientes al segundo período a lo largo del segundo CP (Fig. 3). La única excepción la constituye la muestra correspondiente al primer período temporal del valle inferior del río Chubut, que se agrupa con las muestras pertenecientes al segundo período. En el análisis de las variables canónicas para la región facial (Fig. 4) también puede observarse que las muestras se disponen conformando dos agrupamientos principales a lo largo del segundo eje $(20,20 \%)$. El primero se encuentra conformado por las muestras de Chubut y San Antonio Oeste de ambos períodos, y las muestras correspondientes al segundo período temporal de golfo de San Matías, Isla Gama, valle inferior del río Negro y San Blas. El segundo está constituido por las muestras de laguna del Juncal, valle inferior del río Negro y golfo de San Matías pertenecientes al primer período temporal.

A lo largo del primer CP (CP 1) de la base del cráneo las principales diferencias se observan en el desarrollo lateral y antero-posterior, así como en el tamaño relativo del proceso mastoideo 

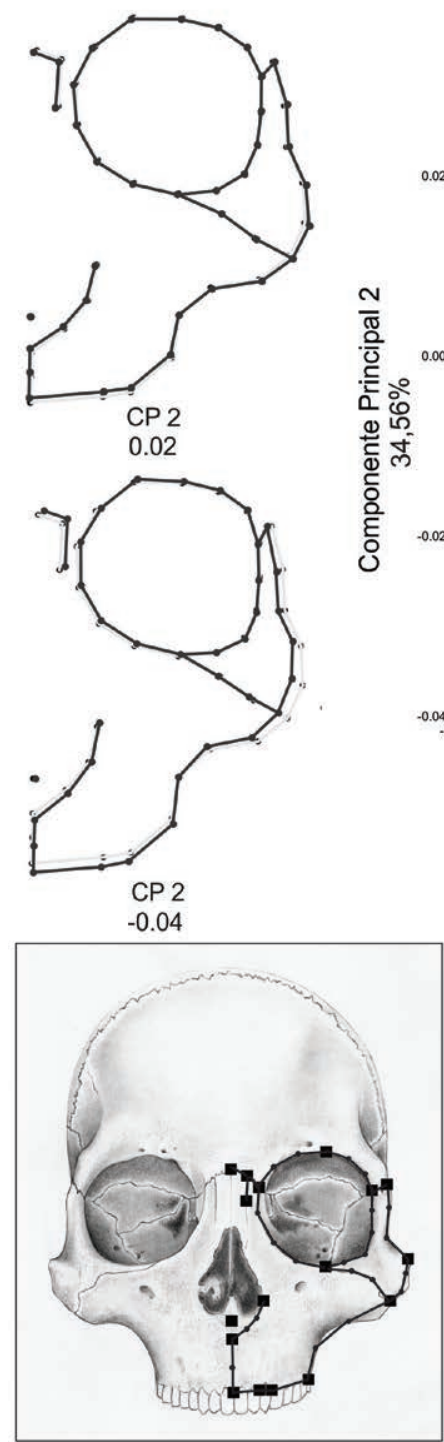

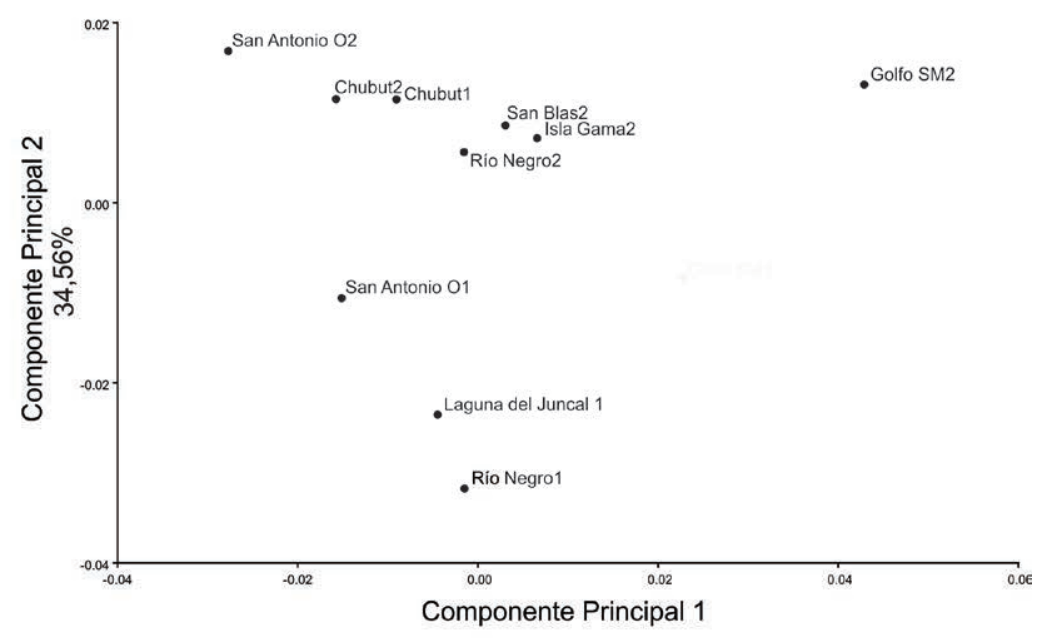

$36,94 \%$
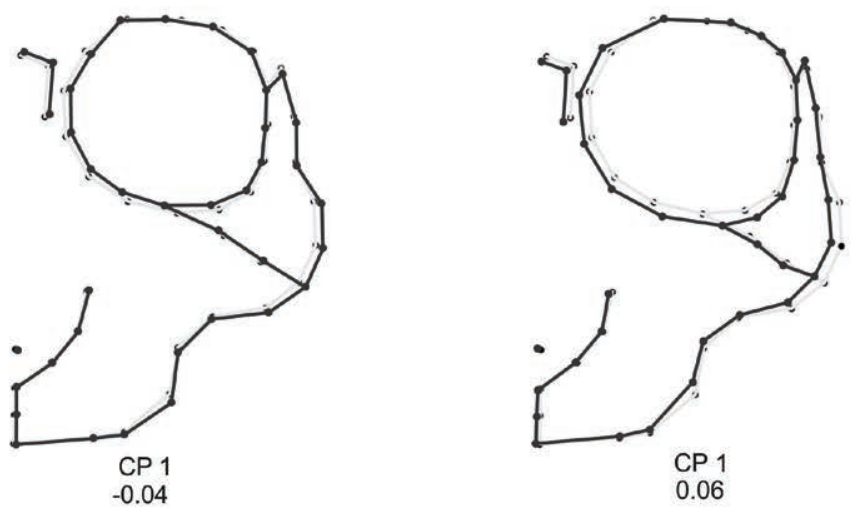

Fig. 3. Análisis de componentes principales (CP) y wireframes de la región facial del cráneo.

(Fig. 5). Las muestras ubicadas en el extremo de los valores negativos presentan un mayor desarrollo antero-posterior de la base del cráneo $y$ del proceso mastoideo, mientras que aquellas correspondientes al extremo positivo poseen un mayor desarrollo lateral de dicho proceso, lo que les confiere una base del cráneo relativamente más ancha. Por otro lado, a lo largo del segundo eje (CP 2), los individuos pertenecientes a las muestras del extremo negativo presentan un foramen magnum de mayor tamaño relativo que aquellos del extremo opuesto. $\mathrm{Al}$ analizar detenidamente los wireframes puede observarse que el proceso mastoideo de las muestras del extremo positivo es más robusto, ya que presentan un desarrollo lateral relativamente mayor en relación con su desarrollo antero-posterior. Finalmente, puede decirse que en términos generales la región basal de los individuos de las muestras del extremo de los valores negativos es relativamente más larga y angosta que la de las muestras del extremo opuesto (Fig. 5).

En el caso del análisis de variables canónicas, para el primer eje (VC 1), la robustez del proceso mastoideo, así como el desarrollo lateral y antero- 

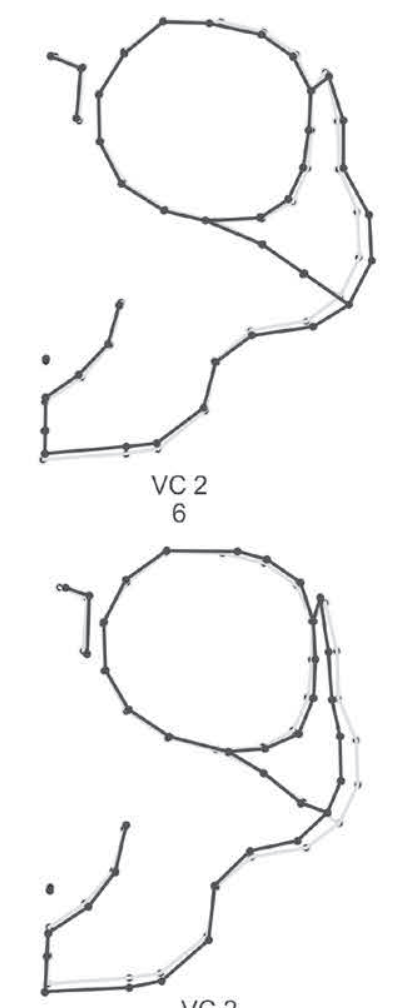

$\mathrm{VC} 2$

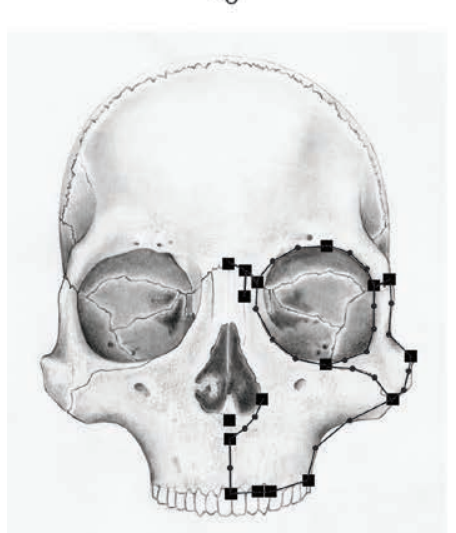

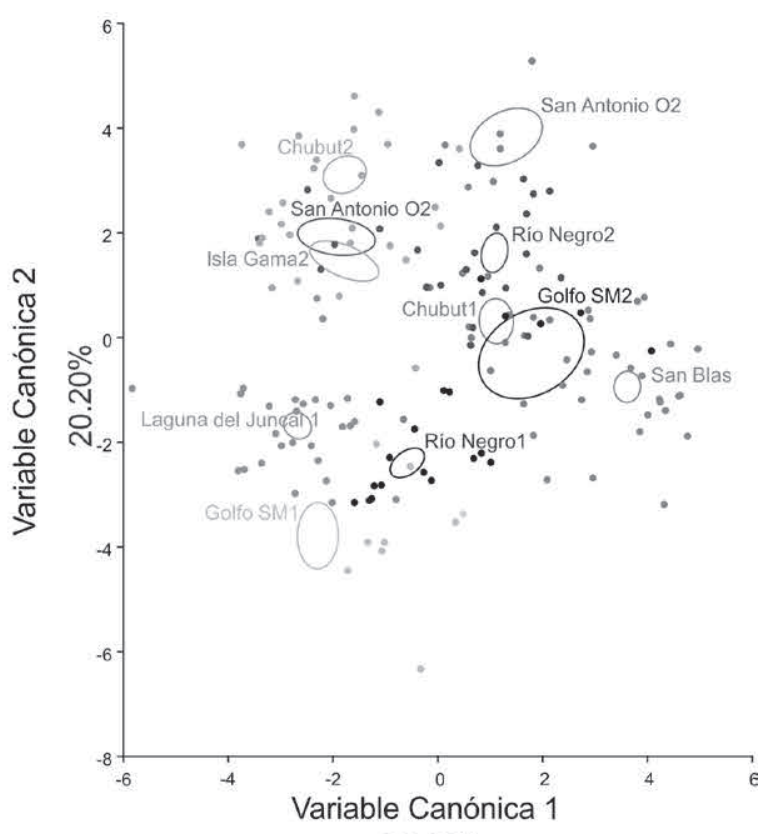

$22.43 \%$
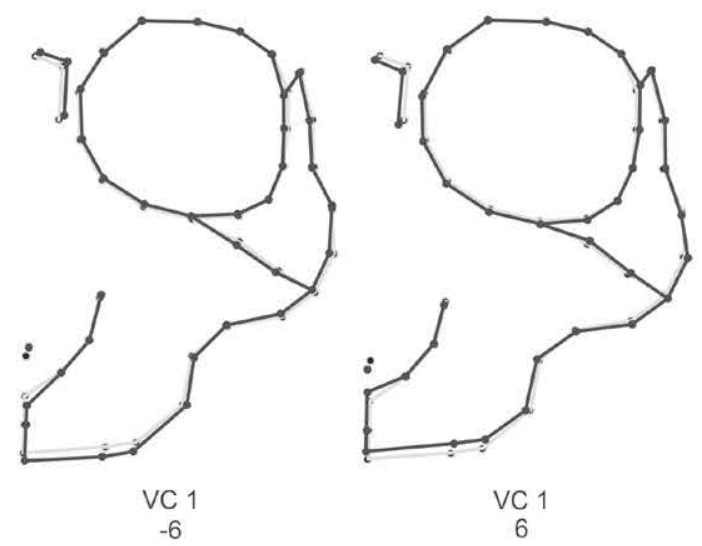

Fig. 4. Análisis de variables canónicas (VC) y wireframes de la región facial del cráneo.

posterior de la región basal del cráneo en su conjunto presenta una tendencia de variación morfológica similar al presentado en el primer $\mathrm{CP}$ (CP 1). Sin embargo, se observan también diferencias en el tamaño relativo del foramen magnum, que presenta un mayor desarrollo en los individuos ubicados hacia el extremo de los valores negativos (Fig. 6). Para el segundo eje (VC 2) es válida la descripción realizada para el CP
2, con la salvedad del desarrollo lateral y anteroposterior del proceso mastoideo, que sigue una tendencia opuesta al observado para el análisis de los componentes principales (Fig. 6).

La distribución de las muestras en los CPs 1 y 2 de la base del cráneo (Fig. 5) es notablemente diferente de aquella observada para el caso de la región facial (Fig. 3). Si bien nuevamente puede observarse cómo las muestras procedentes del golfo 


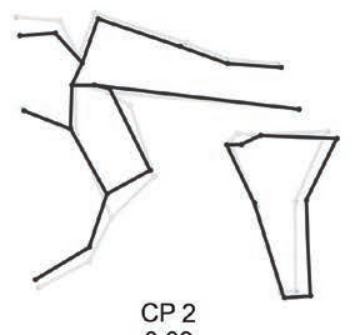

0.09
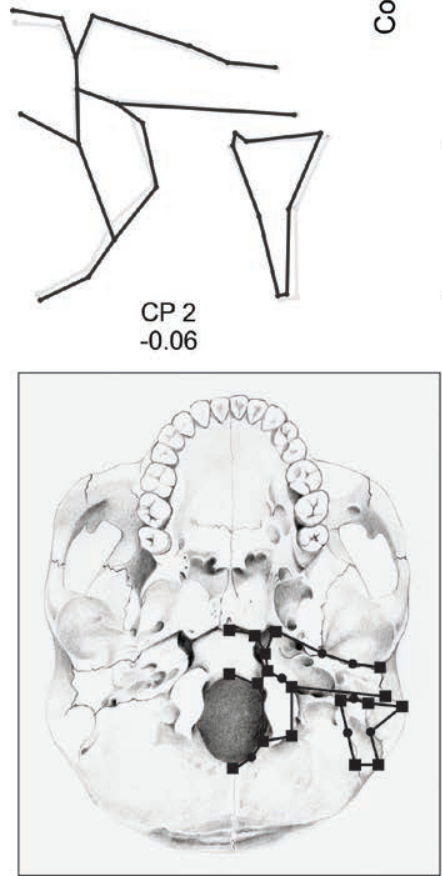

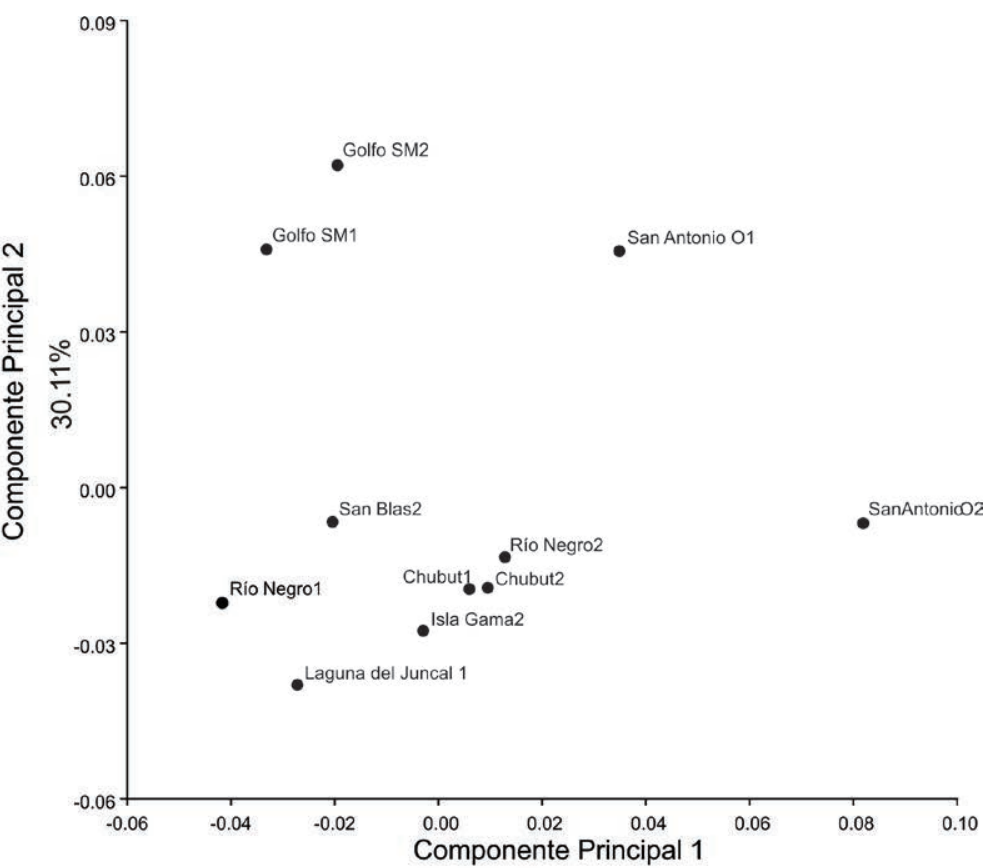

$43.19 \%$

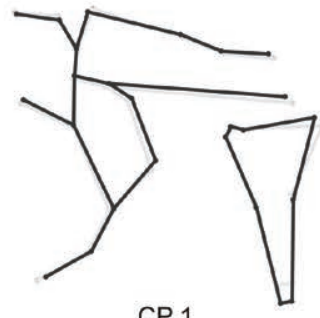

$-0.06$

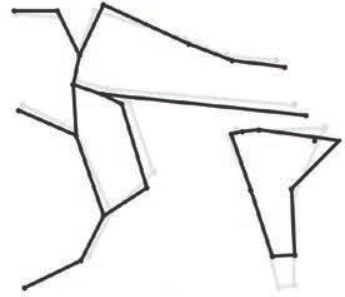

CP 1

Fig. 5. Análisis de componentes principales (CP) y wireframes de la región de la base del cráneo.

de San Matías se alejan del resto de las muestras, resulta llamativo lo próximas que se encuentran entre sí. De esta manera, puede demarcarse una separación entre las muestras procedentes de Isla Gama, San Blas, laguna del Juncal y las del valle inferior del río Negro y del río Chubut de aquellas procedentes de San Antonio Oeste y el golfo de San Matías a lo largo de los dos primeros CPs combinados. En el análisis de las variables canónicas para la región de la base se observa el mismo patrón presentado en los CPs (Fig. 6). Sin embargo, las muestras se agrupan constituyendo dos conjuntos a lo largo de la primera variable canónica (42,13\%): el primero está integrado por las muestras del valle inferior del río Negro y Chubut de ambos períodos, laguna del Juncal, Isla Gama y San Blas; el segundo por las muestras del golfo de San Matías y las de San Antonio Oeste.

Finalmente, el análisis multivariado de la varianza (MANOVA) (Tabla 2), realizado para la región facial empleando los primeros 8 componentes principales (ca. $80 \%$ de la variación) indicó la existencia de diferencias geográficas y temporales significativas en la forma facial media (diferencias entre regiones geográficas: $\mathrm{F}=5,066$; $\mathrm{P}=0,0074$; diferencias temporales: $\mathrm{F}=10,974$; 


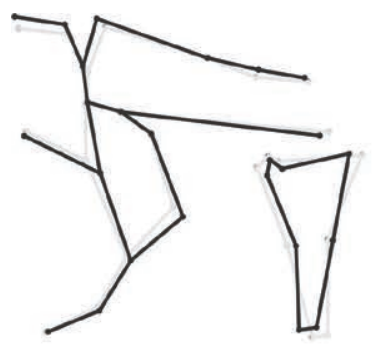

VC 2

6

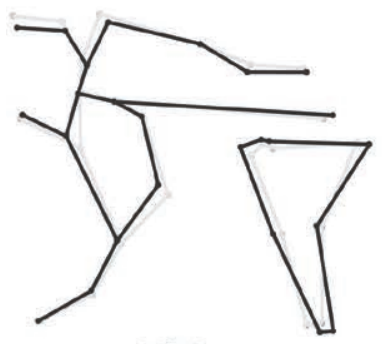

VC 2

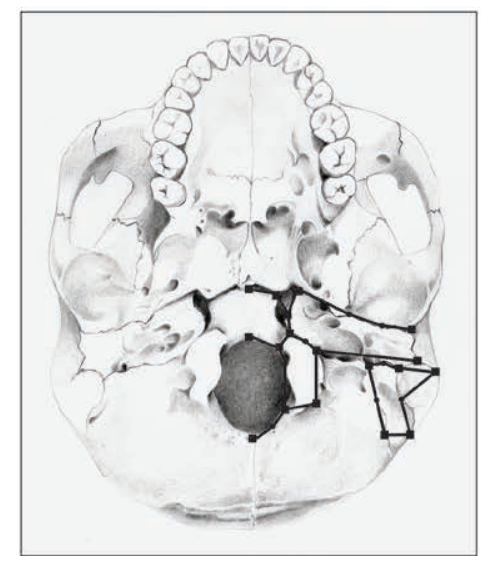

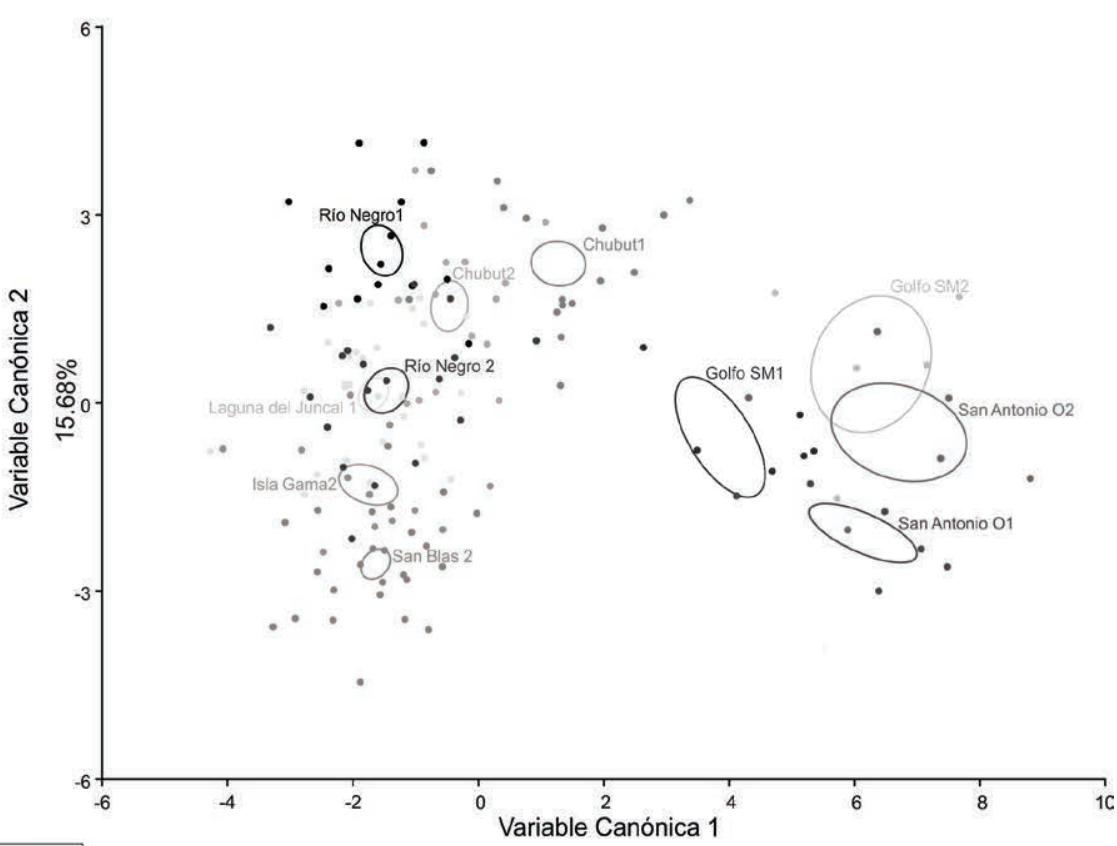

$42.13 \%$

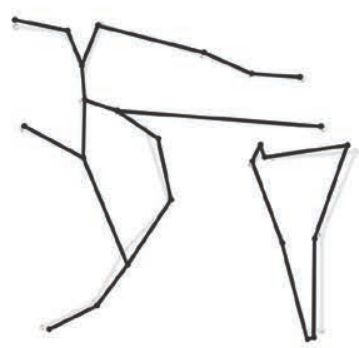

$\mathrm{VC}$
-6

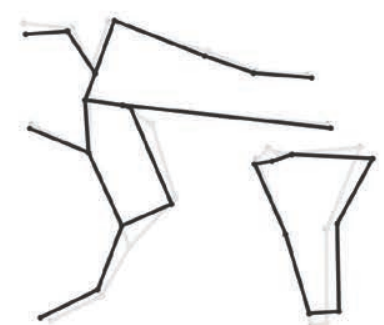

VC 1

Fig. 6. Análisis de variables canónicas (VC) y wireframes de la región de la base del cráneo.

$\mathrm{P}=0,0012)$. En el caso de la región basal, se utilizaron también los primeros 8 componentes principales (ca. $80 \%$ de la variación), siendo los resultados indicativos de diferencias significativas solo para la variación entre regiones geográficas (diferencias entre regiones geográficas: $F=4,731$;

Tabla 2. Resultados del análisis multivariado de la varianza (MANOVA), realizado para la región facial y para la región basal empleando los primeros 8 componentes principales (ca. $80 \%$ de la variación).

\begin{tabular}{cccc}
\cline { 3 - 3 } Región & & Facial & Base \\
\hline \multirow{2}{*}{ Período } & Valor $F$ & 50.662 & 47.312 \\
& $\operatorname{Pr}(>\mathrm{F})$ & $0.007421^{* *}$ & $0.01019^{*}$ \\
\hline \multirow{2}{*}{ Valor $\mathrm{F}$} & 10.974 & 30.526 \\
& $\operatorname{Pr}(>\mathrm{F})$ & $0.001155^{* *}$ & 0.08267 \\
\hline
\end{tabular}

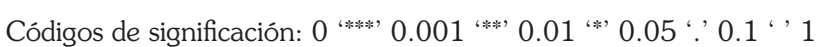


$\mathrm{P}=0,010 ;$ diferencias temporales: $\mathrm{F}=3,053$; $\mathrm{P}=0,083$ ).

\section{DISCUSIÓN}

Nuestros resultados muestran que el patrón de variación morfológica craneofacial observado en el noreste de Patagonia en general, y en el área del golfo de San Matías en particular, es complejo, caracterizado por un comportamiento diferencial de las dos regiones anatómicas estudiadas (el esqueleto facial y la base del cráneo) en relación con la variación ecológica. Los resultados obtenidos para la región facial son concordantes con lo observado en el registro arqueológico, arqueofaunístico y los datos procedentes de los análisis isotópicos en el área de la costa rionegrina del Golfo de San Matías. Estos datos indican que hacia ca. 1.600 años AP se produjo un cambio en la subsistencia, particularmente en la dieta, de las poblaciones humanas prehistóricas que habitaron la región (Favier Dubois et al., 2009; Favier Dubois \& Scartascini, 2012). La tendencia observada en la región del Golfo de San Matías se correspondería con las variaciones que sufrió la morfología costera de la zona. El acceso a recursos costeros críticos como los peces se habría modificado en toda la costa norte del golfo (Favier Dubois et al., 2006; Favier Dubois et al., 2009). En este sentido, los cambios en los escenarios costeros y en el acceso a determinados recursos marinos pudieron haber favorecido la diversificación en las especies consumidas hacia la parte final del Holoceno tardío, a lo que podría sumarse una mayor presión sobre los recursos en vistas de posibles cambios demográficos en esta etapa (Favier Dubois et al., 2009). De este modo, considerando que debido al tiempo de desarrollo relativamente largo del esqueleto facial los factores ecológicos como la composición y dureza de la dieta podrían tener una influencia importante sobre sus cambios morfológicos, particularmente modificaciones en la cantidad relativa de proteínas y la fuerza de mordida (González-José et al., 2005; Perez \& Monteiro, 2009), las diferencias morfométricas observadas entre muestras diacronicas del golfo de San Matías podrían estar relacionadas con procesos ecofenotípicos o de plasticidad fenotípica.

A nivel supra-regional, el patrón de variación facial es concordante con los datos de salud bucal y registro faunístico del Holoceno tardío del curso inferior del río Colorado y áreas vecinas (Flensborg, 2012). Martinez (2008-2009) y Flensborg (2012) plantean que las poblaciones del noreste de Patagonia pueden dividirse en dos bloques temporales considerando la subsistencia, organización tecnológica, movilidad y asentamiento: Holoceno tardío inicial (ca. 3.0001.500 años AP) y Holoceno tardío final (ca. 1.500-250 años AP). Al igual que en la región del golfo de San Matías, hacia el Holoceno tardío final se registraron cambios en el registro faunístico consistentes con una dieta más diversificada, procedente de diversos microambientes (fluvial, marino y terrestre). Bernal, Novellino, Gonzalez y Perez (2007) observaron una marcada tendencia temporal hacia la reducción en las tasas de desgaste dental en las muestras del noreste de Patagonia después del ca. 1.500 años AP. Esto sugiere que las dietas consumidas durante el Holoceno tardío final habrían sido menos duras y/o abrasivas. Bernal et al. (2007) también plantean que estos cambios en la dureza y/o abrasividad de la dieta podrían estar relacionados con la presencia de cerámica en sitios arqueológicos para el Holoceno tardío final.

Los cambios en la morfología facial, principalmente en el componente masticatorio, relacionados con modificaciones en la dieta de poblaciones humanas ya ha sido observado previamente a escala sub-continental y continental (González-José et al., 2005; Perez \& Monteiro, 2009; Perez et al., 2011), sugiriendo que procesos similares han actuado a través de las diferentes escalas espaciales. Una excepción al patrón de diferencias temporales en el noreste de Patagonia la constituyen las muestras procedentes del valle inferior del río Chubut, que se agrupan con las muestras pertenecientes al segundo período temporal. Este resultado concuerda con los de trabajos previos que señalan la existencia de similitudes morfológicas entre las muestras de diferentes periodos temporales provenientes de esta región (Gómez Otero \& Dahinten, 19971998; Perez, 2006). Como fue planteado para las otras regiones estudiadas, este patrón de variación facial concuerda también con las diferencias en el patrón de variación en la subsistencia de la costa de Chubut que muestra que habría habido una 
mayor variabilidad dietaria a lo largo del tiempo, sin poder identificarse las mismas tendencias en la explotación de los recursos de subsistencia que en las áreas vecinas (Gómez-Otero et al., 2007).

Para la base del cráneo los resultados obtenidos indican la existencia de una clara diferenciación entre las muestras de San Antonio Oeste y las del golfo de San Matías con respecto a las demás muestras. En cada región estudiada las muestras pertenecientes al primer período temporal en general se agrupan con las del segundo periodo. Este resultado difiere claramente del obtenido para el esqueleto facial, e indica la existencia de bajos niveles de concordancia entre la base del cráneo y la variación ecológica en el área de estudio. En este sentido la base del cráneo, a diferencia de la región facial, ha sido considerada de particular relevancia para la discusión de la historia evolutiva de las poblaciones humanas (Harvati \& Weaver, 2006; Lockwood et al., 2004; Perez \& Monteiro, 2009). Estos trabajos han sugerido que la base del cráneo presenta un patrón de variación relativamente independiente de la influencia del ambiente y relacionado con procesos aleatorios, por encontrarse sujeta a una menor influencia medioambiental durante la ontogenia que la región facial, ya que es la primera región del cráneo en alcanzar el tamaño y forma adulta (Harvati \& Weaver, 2006; Perez \& Monteiro, 2009). Asimismo, en esta región no se insertan los principales músculos vinculados con la masticación, y por lo tanto no está sometida a las mismas fuerzas biomecánicas que el esqueleto facial. Por lo tanto los resultados obtenidos para la base del cráneo sugieren que los individuos del golfo de San Matías y San Antonio Oeste pertenecientes a diferentes periodos temporales provienen de una única población o linaje en evolución con continuidad temporal en el área. La misma conclusión puede ser esgrimida para las otras regiones estudiadas, reafirmando las diferencias geográficas, más que temporales entre las poblaciones del noreste de Patagonia (Martínez, 2008-2009). Sin embargo, para reforzar esta hipótesis es indispensable el empleo de marcadores moleculares - e.g., ADN mitocondrial (ADNmt) y nuclear - de poblaciones actuales y prehistóricas.

Varios estudios moleculares recientes han sugerido la existencia de continuidad temporal entre las poblaciones de diferentes regiones de Sudamérica y en particular de Patagonia (de Saint Pierre et al., 2012; Figueiro \& Sans 2007; Motti, 2012; Perez et al., 2009). En particular, los estudios efectuados con poblaciones actuales plantean que las variantes actuales de ADNmt (i.e., haplogrupos y haplotipos) presentes en diferentes regiones de Patagonia tienen una ancestría antigua en cada una de las mismas (de Saint Pierre et al., 2012; Motti, 2012), sugiriendo continuidad regional y aislamiento geográfico. Asimismo, los estudios efectuados sobre ADN antiguo señalan que los haplogrupos presentes en las muestras del Holoceno temprano y tardío inicial del noreste de Patagonia y el Sudeste de la Región Pampeana coinciden con aquellos presentes en las poblaciones actuales (Figueiro \& Sans, 2007; Perez et al., 2009). Estos hallazgos apoyan las hipótesis planteadas a partir de la variación actual en el ADNmt y también sugieren que los patrones observados en la base del cráneo son concordantes con la historia de continuidad poblacional a nivel regional.

En conjunto estos resultados difieren de ideas previas que sostuvieron que hacia el Holoceno tardío final (ca. 1.500 años AP) se habría producido una expansión de las poblaciones de la región central de Patagonia (e.g., desembocadura del río Chubut) hacia el noreste de Patagonia y el Sudeste de la región Pampeana, ocasionando un reemplazo poblacional (Barrientos \& Perez, 2002, 2004; Barrientos et al., 2005) o intensificación en el flujo génico (Flensborg, 2012; Martinez, 20082009; Perez, 2006), así como afirmaciones previas acerca de reemplazos poblacionales en el área (Bórmida, 1953-1954). Las diferencias entre los resultados previos y los obtenidos en este trabajo probablemente se deben a que los primeros fueron basados en análisis de la variación morfométrica en el esqueleto facial (Barrientos \& Perez, 2002, 2004). Las similitudes morfométricas en el esqueleto facial entre las muestras del Holoceno tardío final del Sudeste de Pampa y el Noreste de Patagonia que fueron interpretadas como producto de movimientos y reemplazos poblacionales, pueden ser mejor interpretadas con la evidencia actual (tanto morfométrica como del ADNmt) como producto de una respuesta ecofenotípica similar ante un cambio supra-regional en la subsistencia. 
La variación craneofacial ha sido empleada con el fin de estimar relaciones biológicas entre poblaciones humanas de la región del noroeste Patagónico desde mediados del siglo XX (Bórmida, 1953-1954; Barrientos \& Perez, 2002, 2004). En este trabajo se adoptó una aproximación diferente, considerando la evolución de la morfología craneofacial como un proceso complejo en el marco de diferentes escenarios de evolución poblacional y ambiental. Nuestros resultados muestran tendencias diferentes para la evolución de la morfología facial y de la base del cráneo, siendo éstas estructuras que difieren en sus propiedades de respuesta frente al ambiente. Estos resultados señalan la complejidad de la evolución fenotípica y poblacional en el noreste de Patagonia, ligada a cambios ambientales (e.g. cambios en la subsistencia de los grupos) que afectaron los patrones de variación morfológica observados.

\section{AGRADECIMIENTOS}

Agradecemos a Valeria Bernal por los comentarios sobre versiones previas de este trabajo. A Hector Pucciarelli del Museo de La Plata y al personal del Museo Etnográfico de Buenos Aires por permitirnos el acceso a las colecciones bajo su cuidado. Asimismo a Florencia Borella (CONICETINCUAPA) y al equipo de trabajo en la costa del golfo San Matías. El estudio presentado aquí se realizó con el marco de los proyectos PPID/UNLP N02 (2010-2012) y PI 11/N691 (2012-2013). Los fechados y análisis isotópicos para el golfo San Matías fueron subsidiados por los proyectos CONICET (PIP 00756), FONCYT (PICT 38264) y National Geographic Society (CRE Grant 8171).

\section{BIBLIOGRAFÍA}

Adams, D.C., Rohlf, F.J. \& Slice, D.E. (2004). Geometric morphometrics: ten years of progress following the 'revolution'. Italian Journal of Zoology 71:5-16.

Badyaev, A.V. (2005). Stress-induced variation in evolution: from behavioural plasticity to genetic assimitation. Proceedings of the Royal Society 272 (B):877-886.

Barrientos, G. \& Perez, S. I. (2002). La dinámica del poblamiento humano del Sudeste de la Región Pampeana durante el Holoceno. Intersecciones en Antropología 3: 41-54.

Barrientos, G. \& Perez, S. I. (2004). La expansión y dispersión de poblaciones del norte de Patagonia durante el Holoceno tardío: evidencia arqueológica y modelo explicativo. En: Contra viento y marea. Arqueología de Patagonia, M.T. Civalero, P.M. Fernández, A.G. Guráieb compiladores. Instituto Nacional de Antropología y Pensamiento Latinoaméricano, Sociedad Argentina de Antropología. Buenos Aires.

Barrientos, G., Perez, S. I., Bernal, V., Gonzalez, P.N., Béguelin, M. \& Del Papa, M. (2005). Changing views about the local evolution of human populations in the southeastern pampas of Argentina during the Holocene. En: Proceedings of the 5th Annual BABAO Conference, S. Zakrzewski \& M. Clegg compiladores. BAR International Series 1383, Oxford. Great Britain.

Béguelin, M. (2010). Tamaño corporal y temperatura en poblaciones cazadoras recolectoras del Holoceno tardío de Pampa y Patagonia. Revista Argentina de Antropología Biológica 12: 27:36.

Bernal, V. Gonzalez, P.N., Perez, S.I \& Del Papa, M. (2004). Evaluación del error intraobservador en bioarqueología. Intersecciones en Antropología 5:129-140.

Bernal, V., Perez, S.I. \& Gonalez, P.N. (2006). Variation and casual factors of craniofacial robusticity in Patagonian hunter-gatherers from late Holocene. American Journal of Human Biology 18: 748-759.

Bernal, V., Novellino, P., Gonzalez, P.N. \& Perez, S.I. (2007). Role of wild plant foods among Late Holocene huntergatherers from Central and North Patagonia (South America): an approach from dental evidence. American Journal of Physical Anthropology 133:1047-1059.

Bernal, V., Gonzalez, P.N., Perez, S. I. \& Pucciarelli, H.M. (2008). Entierros humanos del noroeste de Patagonia:nuevos fechados radiocarbónicos. Magallania 36: 125-134.

Bernal, V., Perez, S. I., Gonzalez, P.N. \& Diniz-Filho, J.A.F. (2010). Ecological and evolutionary factors in dental morphological diversification among modern human populations from southern South America. Proceedings of Royal Society. Biological Sciences 277(B): 1107-1112.

Bogin, B. \& Rios, L. (2003). Rapid morphological change in living humans: implications for modern human origins. Comparative Biochemistry and Physiology 136 (A):71-84.

Bookstein, F.L. Streissguth, A.P., Sampson, P.D., Connor, P.D. \& Barr, H.M. (2002). Corpus callosum shape and neuropsychological deficits in adult males with heavy fetal alcohol exposure. Neuroimage 15:233-251.

Bórmida, M. (1953-1954). Los antiguos Patagones. Estudio de craneología. Runa 6, 55-96. 
Buikstra, J. \& Ubelaker, D. (1994). Standards for Data Collection from Human Skeletal remains. Arkansas Archaeological Survey Research Series 44.

Caroll, S.P., Hendry, A.P., Reznick, D.N. \& Fox, C.W. (2007). Evolution on ecological time-scales. Functional Ecology 21: 387-39.

Coccilovo, J.A., Varella, H.H., Espoueys, O. \& Standen, V. (2001). El proceso microevolutivo de la población nativa antigua de Arica. Chungara Revista de Antropología Chilena 33:13-20

De Saint Pierre, M., Bravi, M.B., Miotti, S.M.B., Fuku, N., Tanaka, M., Llop, E., Bonatto, S.L. \& Moraga, M. (2012). An Alternative Model for the Early Peopling of Southern South America Revealed by Analyses of Three Mitochondrial DNA Haplogroups. PLOS ONE. 9 (7): 1-12.

Diniz-Filho, J.A.F., Nabout, J.C., Campos Telles, M.P., Soares, T.N. \& Ragel, T.F.L.V.B. (2009). A review of techniques for spatial modeling in geographical, conservation and landscape genetics. Genetics and Molecular Biology 32: 203-211.

Favier Dubois, C.M., Borella, F., Manzi, L.M., Cardillo, M., Lanzellotti, S., Scartascini, F., Carolina, M. \& Borges Vaz, E. (2006). Aproximación regional al registro arqueológico de la costa rionegrina. Arqueología de la Costa Patagónica. Perspectivas Para La Conservación, I. Cruz y M. Caratche editores. UNPA y Secretaría de Cultura de la Provincia de Chubut.

Favier Dubois, C.M., Borella, F., Tykot, R.H. (2009). Explorando tendencias en el uso humano del espacio y los recursos en el litoral rionegrino (Argentina) durante el Holoceno medio y tardío. En: Arqueología de la Patagonia. Una Mirada desde el Último Confín, M. Salemme, F. Santiago, M. Álvarez, E. Piana, M. Vázquez y M. E. Mansur compiladores. Editorial Utopías, Ushuaia.

Favier Dubois, C.M. \& Scartascini, F.L. (2012). Intensive fishery scenarios on the North Patagonian coast (Río Negro, Argentina) during the Mid-Holocene. Quaternary International 256: 62-70.

Figueiro, G. \& Sans, M. (2007). Primeros resultados del análisis de ADN mitocondrial del sitio Arroyo Seco 2, Provincia de Buenos Aires, Argentina. Revista Argentina de Antropología Biológica 9(1): 78.

Flensborg, G.A. 2012. Análisis paleopatológico en el curso inferior del río Colorado (Pcia. de Buenos Aires). Exploración y evaluación del estado de salud de sociedades cazadoras-recolectoras en el Holoceno tardío. (Tesis Doctoral Inédita). Universidad Nacional del Centro de la Provincia de Buenos Aires.
Gómez-Otero, J. \& Dahiten, S.. (1997-1998). Costumbres funerarias $y$ esqueletos humanos: variabilidad $y$ poblamiento en la costa nordeste de la provincia del Chubut (Patagonia Argentina). Relaciones de la Sociedad Argentina de Antropología 22-23:101124.

Gómez-Otero J., Tykot R. \& Falk N. (2007). Isótopos estables, dieta y uso del espacio en la costa atlántica centro-septentrional y el valle inferior del río Chubut (Patagonia, Argentina). En Arqueología de FuegoPatagonia. Levantando piedras, desenterrando huesos... y develando arcanos. Fundación CEQUA, Punta Arenas. 151-161.

González-José, R.F., Ramírez-Rozzi, M., Sardi, M., MartínezAbadías, N., Hernández, M. \& Pucciarrelli, H.M. (2005). Functional-cranial approach to the influence of economic strategy on skull morphology. American Journal of Physical Anthropology. 128: 757-77.

Harvati, K. \& Weaver, T.D. (2006). Human cranial anatomy and the differential preservation of population history and climate signatures. The Anatomical Record. 288(A): 1225-1233.

Katzmarzyk, P.T. \& Leonard, W.R. (1998). Climatic influences on human body size and proportions: ecological adaptations and secular trends. American Journal of Physical Anthropology. 106: 483-503.

Klingenberg, C.P. (2011). MorphoJ: an integrated software package for geometric morphometrics. Molecular Ecology Resources 11: 353-357.

Lockwood, C.A., Kimbel, W.H. \& Lynch, J.M. (2004). Morphometrics and hominoid phylogeny: Support for a chimpanzee-human clade and differentiation among great ape subspecies. Harvard University, Cambridge, MA. 13 (101): 4356-4360.

Martínez, G. (2008-2009). Arqueología del curso inferior del río Colorado: estado actual del conocimiento e implicaciones para la dinámica poblacional de cazadores-recolectores pampeano-patagónicos. Cazadores recolectores del cono-sur. Revista de Arqueología. 3, 71-92.

Mitterorcker, P.Y. \& Gunz, P. (2009). Advances in Geometric Morphometrics. Journal of Evolutionary Biology. 36:235-247.

Miotti, J.M.B. (2012). Caracterización de linajes maternos en la población actual del Noroeste y Centro-Oeste argentinos. (Tesis Doctoral Inédita). Universidad Nacional de La Plata.

Nijhout, H.F. (2003). The control of growth. Development. $130,5863-5867$. 
Perez, S.I. (2006). Influencia de la deformación artificial del cráneo sobre la morfología facial: Implicancias para los estudios de relaciones evolutivas inter-poblacionales. Revista Argentina de Antropología Biológica. 8(1): 37-55.

Perez, S.I. (2007). Artificial cranial deformation in South America: A geometric morphometrics approximation. Journal of Archaeological Science. 34: 1649-1658.

Perez, S.I. \& Monteiro, L.R. (2009). Non-random factors in modern human morphological diversification: a study of craniofacial variation in southern South American populations. Evolution. 63: 978-993.

Perez, S.I., Bernal, V. \& Gonzalez, P.N. (2006). Differences between sliding semi-landmarks methods, with an application to human craniofacial and dental variation. Journal of Anatomy. 208: 769-784.

Perez, S.I., Bernal, V., Gonzalez, P.N., Sardi, M. \& Politis, G.G. (2009). Discrepancy between cranial and DNA data of early americans: Implications for american peopling. PLOS ONE. 4(5): e5746.

Perez, S.I., Lema, V. Diniz-Filho, Bernal, V., Gonzalez, P.N., Gobbo, D. \& Pucciarelli, H.M. (2011). The role of diet and temperature in shaping cranial diversification of South American human populations: an approach based on spatial regression and rate tests. Journal of Biogeography. 38:148-163.

Piglucci, M. (2001). Phenotypic Plasticity: Beyond Nature and Nurture. Baltimore, MD, USA: Johns Hopkins University Press.

Pucciarelli, H.M. \& Oyhenart, E.E. (1987). Effects of maternal food restriction during lactation on craniofacial growth in weanling rats. American Journal of Physical Anthropology 72: 67-75.

R Development Core Team . (2012). R: a language and environment for statistical computing. Vienna: $\mathrm{R}$ Foundation for Statistical Computing. Available at: http://www.r-project.org

Reznick, D.N., Shaw, F.H., Rodd, F.H. \& Shaw, R.G. (1997). Evaluation of the rate of evolution in natural populations of guppies (Poecilia reticlrlatri). Science. 275:19341937.

Rohlf, F.J. \& Slice ,D.E. (1990). Extensions of the Procrustes Method for the optimal superimposition of landmarks. Systematic Zoology. 39:40-59.

Rohlf, F.J. (2012). Programas serie TPS. Disponibles en http:// life.bio.sunysb.edu/ee/rohlf/software.html

Roseman, C.C. (2004). Detecting interregionally diversifying natural selection on modern human cranial form by using matched molecular and morphometric data. Proceedings of the Natlional Academy of Sciences. 101:12824-12829.

Rothammer, F. \& Silva, C. (1990). Craniometrical variation among South American prehistoric populations: climatic, altitudinal, chronological and geographic contributions. American Journal of Physical Anthropology 82:9-17.

Sardi, M.L. \& Ramirez-Rozzi. (2005). A cross-sectional study of human craniofacial growth. Annals of Human Biology. 32:390-396.

Scartascini, F. Charo, M. \& Volpedo, A. (2009). Caracterización de las estrategias de obtención de recursos ícticos a partir del análisis de otolitos. El caso de la costa norte el golfo de San Matías (Río Negro). En: Arqueología de Patagonia: una mirada desde el último confín, M. Salemme, F. Santiago, M. Álvarez, E. Piana, M. Vázquez y E. Mansur editores. Utopías. Ushuaia.

Schluter, D. (2000). The ecology of adaptive radiation. Oxford University Press, New York.

West-Eberhard, M.J. (2003). Developmental Plasticity and Evolution. New York: Oxford University Press. 
